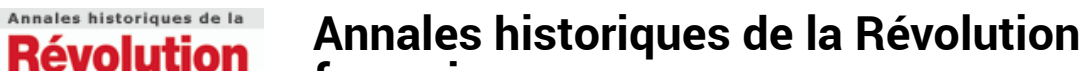

française française

324 | avril-juin 2001

Louis Charles Antoine Desaix. Officier du roi, Général de la République

\section{La Légende de Desaix}

Dans les mémoires de deux de ses aides de camp et dans les écrits de Sainte-Hélène

\section{Thierry Lentz}

\section{(2) OpenEdition}

\section{Journals}

Édition électronique

URL : https://journals.openedition.org/ahrf/411

DOI : 10.4000/ahrf.411

ISSN : 1952-403X

Éditeur :

Armand Colin, Société des études robespierristes

Édition imprimée

Date de publication : 1 juin 2001

Pagination : 151-159

ISSN : 0003-4436

Référence électronique

Thierry Lentz, "La Légende de Desaix », Annales historiques de la Révolution française [En ligne], 324 avril-juin 2001, mis en ligne le 22 mai 2006, consulté le 23 avril 2022. URL : http://

journals.openedition.org/ahrf/411; DOI : https://doi.org/10.4000/ahrf.411

Ce document a été généré automatiquement le 23 avril 2022

Tous droits réservés 


\section{La Légende de Desaix}

Dans les mémoires de deux de ses aides de camp et dans les écrits de Sainte-Hélène

\section{Thierry Lentz}

1 Avec des généraux tels Kléber, Leclerc ou Richepance, Desaix fut un des "grands morts» du Consulat. Le régime put le mettre en valeur sans crainte de favoriser l'émergence d'un rival à Bonaparte, et pour cause. L'image du personnage en a été comme lissée, jusqu'à gommer tout doute, toute critique, voire toute analyse équilibrée de sa carrière. Desaix devint très vite une sorte de " héros » idéal : il avait donné sa vie pour sauver le régime consulaire et son chef. Il l'avait fait presque de gaieté de cœur car, nous dit-on, il admirait Bonaparte envers qui il fit preuve d'une fidélité à toute épreuve. Comme en échange de ce sacrifice, les dieux lui avaient offert une mort sans douleur et ouvert la voie d'une belle postérité. Quant à Napoléon, conscient du sacrifice de son «ami », il ne s'était pas senti autorisé à le pleurer mais avait bien mesuré la grandeur et l'utilité du sacrifice. On lui élèverait donc des statues, on chanterait ses louanges, on le rendrait seul artisan de la victoire de Marengo, jusqu'à en faire un héros un peu fade, ce qu'un auteur contemporain, fondateur de l'école des nouveaux romantiques, a parfaitement reproduit dans une biographie récente ${ }^{1}$.

2 Ce portrait du «Desaix idéal » a de nombreuses sources. On le rencontre notamment dans la série de mémoires que nous avons examinés pour dégager le portrait postmortem de Desaix par ceux qui l'ont « connu et aimé ». Ces témoignages participent à la création de l'image d'un général qui ne semble être venu sur terre que pour offrir sa fidélité et sa vie au héros napoléonien.

3 Ainsi se poursuit à Sainte-Hélène - où Napoléon occupe son exil à sculpter sa légende en jugeant sa vie, son œuvre et ses contemporains - la création d'un Desaix héroïque et désintéressé. Même tonalité dans les mémoires des aides de camp du "Sultan Juste ", Rapp et Savary, deux militaires qui l'ont connu, servi et aimé.

Quelles sont les grandes lignes de ce portrait? Cette communication tentera de la dire, sans ouvrir de débat sur les faits, sur l'exactitude du témoignage, mais en se concentrant sur le portrait moral du général Desaix, tel qu'il fut popularisé par ces différents mémoires. 
Les mémoires de Rapp et Savary

5 Parmi les aides de camp de Desaix, les deux plus connus sont Rapp et Savary. L'un et l'autre entrèrent à son service à l'armée de Sambre-et-Meuse. Ils le servirent donc pendant environ cinq ans et assistèrent à tous les épisodes de sa carrière proprement «napoléonienne », en Italie, en Égypte et à Marengo. Nouvelle preuve de l'affection qui unissait Napoléon à Desaix, le Premier consul les prit à son service quelques jours après la mort de leur chef.

6 Leurs mémoires sont, pour le sujet qui nous intéresse, de valeur inégale. Cela tient à la différence des deux personnalités. Militaire courageux, Rapp s'illustra sur tous les champs de bataille de l'Empire ${ }^{2}$. Il fut un des généraux les plus souvent blessés. Savary était tout l'inverse. Beaucoup plus « politique » que son collègue, il connut une carrière militaire moins brillante (même s'il commanda en chef en Pologne et en Espagne), fondant surtout sa réputation sur les coups tordus qu'il exécuta sur ordre de Napoléon. Son savoir-faire en la matière lui valut même de succéder à Fouché au ministère de la Police générale où il passa quatre années ${ }^{3}$.

7 Les mémoires du général Rapp ont été publiés dès 1823, chez le célèbre libraire Bossange, en un volume de 439 pages $^{4}$. Ils ont été réédités et annotés en 1895 par Désiré Lacroix. Une troisième publication, en réalité un fac-similé de l'édition originale, a été donnée en 1973 par ses descendants. Ces mémoires sont synthétiques et contiennent en réalité peu de détails sur l'époque où Rapp fut aide de camp de Desaix. Le texte s'ouvre pourtant sur cette période, mais enchaîne rapidement sur un portrait de Napoléon. Les souvenirs de Rapp ne deviennent plus conséquents qu'à partir de la bataille d'Austerlitz. Au départ, ce texte ne devait pas être publié tel quel : le décès de Rapp, en 1821, en accéléra la sortie. L'ouvrage n'était pas achevé. Quelque «tinturier» (ces hommes de plume qui aidaient les témoins à écrire leurs mémoires et leur suggéraient souvent des «arrangements» avec les faits pour faire mieux vendre) l'aurait sans doute amélioré.

8 Si l'intérêt général des Mémoires de Rapp est faible, l'authenticité des faits relatés est presque garantie. Hélas, Desaix n'est guère présent que dans les quatre premières pages du premier chapitre. Rapp raconte comment, jeune lieutenant, il fut remarqué par le général qui l'appela auprès de lui comme aide de camp après la bataille d'offenburg dont, dit-il, Desaix avait été le «modeste vainqueur ». En une cinquantaine de ligne, toute sa collaboration avec ce premier chef est passée en revue. Tout juste si Desaix a droit au qualificatif de «brave » au moment de la narration - en une demi-phrase - de sa mort à Marengo. Cependant, Rapp nous donne l'indice de l'affection qu'éprouvait Bonaparte pour le héros trop tôt disparu : «Le Premier consul daigna m'attacher à sa personne ; j'héritai de sa bienveillance pour le conquérant de la haute Égypte $»^{5}$. S'il avait eu plus de temps pour peaufiner ses mémoires, Rapp aurait peut-être mieux décrit Desaix dont la mort, survenue en même temps que celle de son père, l'avait profondément abattu. Juste après Marengo, il avait écrit à sa mère : "Le sort n'a pas cessé de m'accabler et s'est ingénié à augmenter ma douleur par la mort de mon malheureux général [...] Je n'oublierai jamais mon malheureux ami et bienfaiteur Desaix $»^{6}$. À la lecture de ses mémoires inachevés, il n'eut pas le temps de tenir cette promesse.

9 Desaix est bien plus présent dans les mémoires de Savary où la collaboration de l'auteur avec lui occupe les trois quarts du premier volume. Les mémoires de Savary font partie des grands témoignages sur le Consulat et l'Empire. Ils furent publiés chez Bossange, en 
18287. Ces huit volumes eurent, nous dit Jean Tulard, un «rententissement considérable $»^{8}$ tant en raison de la personnalité et de la carrière de leur auteur que pour leur contenu. Ils servirent surtout à Savary de justification dans différentes affaires sensibles auxquelles il fut mêlé : duc d'Enghien, mort de Pichegru, affaire Malet, etc. S'il se fit aider pour les rédiger, l'étude de fragments disponibles aux Archives de la Guerre ${ }^{9}$ montre que sur les épisodes qui nous intéressent, il n'a maquillé ni les faits, ni ses sentiments.

Comme Rapp, Savary semble avoir beaucoup aimé Desaix. Il en donne toujours une image des plus favorables. Réciproquement, Napoléon avait pour Desaix une " haute estime » et " de l'amitié $»^{10}$. Réciproquement, Savary présente Desaix comme étant, lors de son séjour à l'armée d'Allemagne, « un grand admirateur de la gloire (du général Bonaparte) ", ajoutant que son général " appelait de ses vœux secrets un homme de caractère et de génie qui eut l'audace de se placer au timon des affaires, qui put comprimer l'anarchie, et rendre à l'armée le lustre que lui méritaient ses services ». Et Savary de conclure: «Le vainqueur d'Arcole lui semblait seul pouvoir être cet homme $»^{11}$. Ainsi, Bonaparte et Desaix étaient faits pour s'entendre, ce dernier ayant d'emblée conçu son rôle comme celui d'un loyal second.

11 En Italie, où les deux hommes attendent de s'embarquer, puis en Égypte, Savary nous montre un Desaix « avide de connaissances ", actif dans les préparatifs et décidé dans l'action. Il est une sorte de héros romantique avant de devenir le «Sultan juste » : on le voit s'activer lors du débarquement devant Alexandrie, offrir sa monture pour qu'elle soit attelée à une pièce d'artillerie qui manque de chevaux de trait, interdisant tout pillage y compris lorsque l'armée harassée manque de tout ${ }^{12}$. L'aide de camp nous révèle même l'humanité de son chef dans cette campagne qui en fut souvent dénuée : «Le général Desaix aimait beaucoup les Turcs et souvent il priait le docteur Renoult de donner des soins à ceux d'entre eux dont l'influence et le crédit lui étaient nécessaires $»^{13}$.

Lorsqu'il narre les négociations d'El-Arich, auxquelles il participa aux côtés de Desaix, Savary prend parti pour son chef contre le général Kléber qu'il juge trop pressé d'en finir afin de rentrer en France. Il remarque cependant que Desaix excellait plus dans le commandement que dans la diplomatie.

13 Suivant son général lors de son retour en Europe, Savary l'assiste encore lors de la campagne d'Italie. Il sert d'agent de liaison entre Desaix et Bonaparte à Marengo. C'est encore Savary qui retrouve le corps de Desaix sur le champ de bataille à la tombée de la nuit $^{14}$. Il tente toutefois de rétablir l'équilibre et la vérité entre les mérites de Desaix et ceux de Kellermann qui, sans doute, prit une part décisive au succès. Napoléon luimême ne disait-il pas : « Le petit Kellermann a donné bien à propos » ?

Comme Rapp, la mort de Desaix plonge Savary dans la tristesse... qui s'efface promptement lorsqu'il apprend qu'il devient aide de camp du Premier consul. À partir de ce moment, la carrière du futur duc de Rovigo est assurée.

Les écrits de Sainte-Hélène

15 Nous sommes le 2 mai 1821. Au centre de l'Atlantique Sud, sur la petite île anglaise de Sainte-Hélène. Dans trois jours, Napoléon sera mort. Antommarchi, dernier médecin de l'Empereur est à son chevet. Dans ses mémoires, il raconte: «Deux heures après midi. La fièvre redouble. Délire. L'Empereur ne parle que de la France, de son fils, de ses compagnons d'armes. Steingel, Desaix, Masséna! Ah! La victoire se décide; allez, courez, pressez la charge; ils sont à nous $»^{15}$. On recherchera en vain confirmation de ce 
témoignage dans les écrits du valet de chambre Marchand ou ceux du grand-maréchal Bertrand, autres témoins de la scène. Ni l'un ni l'autre ne rendent comptent qu'au milieu de son délire, Napoléon appela une dernière fois Desaix.

Peu importe au fond dans le cadre qui est le nôtre. Ce que nous constatons, c'est que témoignages authentiques ou soigneusement réécrits -, les écrits de Sainte-Hélène poursuivent la construction du mythe de Desaix, qu'il s'agisse du Napoléon dans l'exil d'O'Meara, paru dès 1822, du Mémorial de Sainte-Hélène, paru en 1823, du Journal de Gourgaud, publié pour la première fois en 1899 ou, à un degré moindre (car trop récemment découverts pour avoir contribué à l'entretien du mythe), des Cahiers de Sainte-Hélène de Bertrand, déchiffrés et publiés seulement entre 1949 et 1959.

Dans ces principaux « évangiles » (Jean Tulard), comme dans d'autres écrits ou dictées de Sainte-Hélène, on ne trouvera pas un mot défavorable à Desaix. En une seule occasion, Gourgaud émet une ombre de critique pour défendre Kléber, souvent opposé au conquérant de la haute Égypte. Mais elle est discrète, n'émane pas directement de l'Empereur (mais de celui qui converse avec lui) et n'a donc que peu de conséquences sur le portrait de Desaix.

Les écrits de Sainte-Hélène ont sans le moindre doute contribué au renforcement de la légende de Desaix, créée sous le Consulat. Ils dressent un portrait moral (à tous les sens du terme) du général. C'est d'autant plus remarquable que le "tribunal de SainteHélène ", devant lequel comparurent les acteurs de l'épopée, rendit de sévères jugements, y compris là où on ne s'y attendait pas. Pendant ces années de captivité, Napoléon ressassa ses rancunes et entreprit d'offrir à la postérité, non seulement le compte rendu de ses actes, mais encore son opinion sur les hommes qui l'entourèrent. Dans ce jeu de massacre, Desaix apparait comme un des mieux lotis.

19 Lorsqu'il parle de ce général à ses compagnons, Napoléon le compare souvent aux autres généraux de la Révolution et, singulièrement, à Kléber. «L'Empereur répétait à satiété que l'Égypte devait demeurer à la France et qu'elle y fût infailliblement demeurée si elle eût été défendue par Kléber ou Desaix », peut-on lire dans le Mémorial. Et, plus loin, il compare les deux hommes : «C'étaient ses deux grands lieutenants les plus distigués, disait-il ; tous deux d'un grand et rare mérite, quoique d'un caractère et de dispositions bien différents. Kléber était le talent de la nature : celui de Desaix était entièrement celui de l'éducation et du travail. Le génie de Kléber ne jaillissait que par moments, quand il était réveillé par l'importance de l'occasion, et il se rendormait aussitôt après au sein de la mollesse et des plaisirs. Le talent de Desaix était de tous les instants ; il ne vivait, ne respirait que l'ambition noble et la véritable gloire : c'était un caractère tout à fait antique $»^{16}$. La comparaison avec Kléber est encore reprise dans le Journal de Gourgaud: "(Kléber) ne songeait qu'aux femmes et aux amusements de la capitale. Il n'aimait la gloire que comme le chemin des jouissances, tandis que Desaix aimait la goire pour la gloire. [...] Desaix était un tout autre homme ; si je l'avais laissé en Égypte, j'aurais conservé ma conquête $»^{17}$. C'est ce jour-là que Gourgaud prend la défense de Kléber. Selon lui, Desaix n'avait pas commandé en chef avant la campagne d'Égypte tandis que Kléber a commandé l'armée du Rhin. Le mérite du second ne devait donc pas être sous-estimé. Napoléon le reconnaît et concède que la guerre contre les Turcs n'était « rien » comparée à ce que fit Kléber sur le Rhin.

20 Si l'on en croit les confidences de l'Empereur déchu, Desaix et lui étaient faits pour s'entendre... et ce d'autant que le premier ne contesta jamais la prééminence du second. Selon Napoléon, en effet, lorsque, général en chef de l'armée d'Italie, il 
rencontra Desaix, celui-ci avait déjà pour lui « la plus vive admiration ${ }^{18}$. Quant à lui, il reconnut immédiatement la valeur de ce général venu de l'armée du Rhin et voulut se l'attacher. Desaix accepta et, fut, dès cet instant, l'élève de Bonaparte : «Desaix m'a souvent assuré en Égypte qu'avant d'avoir servi sous moi, il n'avait aucune idée de la guerre ", confia Napoléon au général Gourgaud ${ }^{19}$. Une fois encore apparaît le «donnant-donnant» de la légende de Desaix : il était donc d'autant plus grand qu'il reconnaissait la valeur supérieure de Napoléon. Ce dernier appréciait tant son subordonné qu'il envisagea, nous dit-il, de lui donner la main de Désirée Clary, son ancienne fiancée, main sur laquelle il avait si peu de droits que l'intéressée se maria, et sans lui demander son avis, avec le général Bernadotte ${ }^{20}$. Il fallait que Desaix fût un homme de grande valeur pour avoir le droit de succéder à Napoléon dans les bras de Désirée.

21 Selon Napoléon, Desaix était, avec Marceau, Pichegru, Hoche et Kléber, un des meilleurs produits de la Révolution ${ }^{21}$. Mais alors que Pichegru devint son ennemi (et mourut dans des conditions suspectes au Temple), que Marceau disparut très vite de la scène militaire et politique, que sa rivalité avec Hoche s'acheva avec la mort de celui-ci, que Kléber lui en voulait beaucoup d'avoir abandonné l'armée en Égypte, il n'avait rien à craindre de Desaix. En Égypte, alors que tous le généraux maugréaient contre les difficultés de la campagne, «Desaix seul pensait comme moi $»^{22}$ confia l'Empereur à Gourgaud.

Dans la relation des campagnes d'Égypte et de Syrie qu'il dicta à ses compagnons d'exil et qui constitue une partie des "mémoires » de Napoléon, le portrait de Desaix n'est pas moins flatteur. Lorsque Napoléon raconte la conquête de la haute Égypte, il explique pourquoi son choix se porta sur Desaix pour commander l'expédition: «Personne n'était plus propre à diriger une pareille opération que Desaix ; personne ne le désirait avec plus d'ardeur. Jeune, la guerre était sa passion ; insatiable de gloire, il connaissait toute celle qui était attachée à la conquête de ce berceau des arts et des sciences $»^{23}$.

Las Cases écrit encore : «L'Empereur dit que sa mort a été la plus grande perte qu'il ait pu faire; leur conformité d'éducation et de principes eussent fait qu'ils se seraient toujours entendus ; Desaix se serait contenté du second rang, et fût toujours demeuré dévoué et fidèle. S'il n'eût pas été tué à Marengo, le Premier consul lui eût donné l'armée d'Allemagne, au lieu de la confier à Moreau " $^{24}$. "Un grand général n'est pas une chose ordinaire, confiait un autre jour l'Empereur à Gourgaud; de tous les généraux de la Révolution, je ne connais que Desaix et Hoche qui eussent pu aller loin $»^{25}$.

24 La mort de Desaix fut donc, selon Napoléon, un grand drame qui le privait d'un de ses meilleurs subordonnés. On relèvera, pour le plaisir de relever une des (nombreuses) erreurs de Las Cases, que le mémorialiste lui fait dire que Desaix fut tué « d'un coup de canon $»^{26}$. À O’Meara, il déclara : « Kléber et Desaix furent deux pertes irréparables pour la France $\aleph^{27}$. Il se dit en outre fort affecté par des rumeurs le soupçonnant d'avoir fait tuer Desaix à Marengo : « Hoche périt subitement et avec des circonstances singulières qui donnèrent lieu à beaucoup de conjectures; et comme il existait un parti avec lequel tous les crimes me revenaient de droit, l'on essaya de répandre que je l'avais fait empoisonner. Il fut un temps où rien de mauvais ne pouvait arriver que je n'en fusse l'auteur ; ainsi, de Paris, je faisais assassiner Kléber en Égypte ; à Marengo, je brûlais la cervelle à Desaix $»^{28}$. 

transformer en général à l'antique. Pour avoir été digne d'une telle admiration, d'une telle fidélité, d'une telle amitié et d'un tel sacrifice, Bonaparte ne pouvait être qu'un homme hors du commun.

\section{NOTES}

1.Gonzague Saint-Bris, Desaix, le sultan juste, Paris, Perrin, 1995, 220 pages.

2.Sur Rapp : Philippe Jéhin, Rapp, le sabreur de Napoléon, Strasbourg, La Nuée Bleue, 1999, 287 pages.

3.Sur Savary : Thierry Lentz, Savary, le séide de Napoléon, Metz, Serpenoise, 1993, 321 pages.

4.Mémoires du général Rapp (1772-1821), aide de camp de Napoléon, écrits par lui-même, Paris, Bossange, 1823, 439 pages. Pour cet article, nous utiliserons l'édition de 1895, revue et annotée par Désirée Lacroix (Garnier éditeur, 464 pages).

5.Ibidem, p. 4.

6.Lettre citée par P. Jéhin, Rapp, le sabreur de Napoléon, p. 78. 
7.Mémoires du duc de Rovigo pour servir à l'histoire de l'empereur Napoléon, Paris, Bossange, 1828, 8 volumes. Une réédition en cinq volumes a été publiée chez Garnier en 1900. Nous travaillons ici sur l'édition de 1828.

8.Jean Tulard, Nouvelle bibliographie critique des mémoires sur l'époque napoléonienne écrits ou traduits en français, Genèvre, Droz, 1991, p. 267.

9.Voir le Journal autographe du chef d'escadron Savary, aide de camp du général Desaix, depuis le départ de Malte jusqu'au retour du général Desaix en France que nous avons retrouvé, au S.H.A.T., dans les papiers du général Belliard (MR 544).

10.Mémoires du duc de Rovigo, I, p. 176.

11.Ibidem, I, p. 20.

12.Ibidem, I, pp. 50-97.

13.Ibidem, I, p. 131. La remarque de Savary signifie-t-elle, a contrario, que Desaix refusait son aide à ceux qui ne lui étaient pas « nécessaires »?

14.Ibidem, I, pp. 283-284.

15.Cité par Jean Tulard, Napoléon à Sainte-Hélène, Paris, Robert Laffont, 1981, p. 731.

16.Comte Emmanuel de Las Cases, Le Mémorial de Sainte-Hélène, édition critique de Marcel Dunan, Paris, Flammarion, 1951, t. I, p. 172.

17.Général baron Gougaud, Journal de Sainte-Hélène. 1815-1818, préface et notes d'Octave Aubry, Paris, Flammarion, 1947, t. II, p. 173.

18.Le Mémorial de Sainte-Hélène, II, p. 427. Ici, Napoléon et ses aides de camp, notamment Savary, se rejoignent. Mais le duc de Rovigo écrivit ses Mémoires plusieurs années après la parution du Mémorial. Se considérant lui-même comme le "séide » de Napoléon, n'aurait-il pas fait en sorte que son témoignage confirme point par point celui de son ancien maître ? La question doit au moins être posée. Une réponse positive renforcerait d'ailleurs l'hypothèse de la création volontaire de la légende « romantique » de Desaix par Napoléon.

19.Journal de Sainte-Hélène, II, p. 51.

20.Général Bertrand, Cahiers de Sainte-Hélène. Janvier-mai 1821, déchiffrés par Paul Fleuriot de Langle, Paris, Albin Michel, 1959, p. 42.

21.Le Mémorial de Sainte-Hélène, II, p. 576.

22.Journal de Sainte-Hélène. 1815-1818, I, p. 244. Cette information est infirmée par certaines lettres de Desaix à ses aides de camp, citées, lors du colloque de Riom, par M. Philippe-Jean Vidal.

23. «Campagne d'Égypte et de Syrie », Correspondance de Napoléon 1er, t. XXIX, p. 517.

24.Le Mémorial de Sainte-Hélène, I, p. 173.

25.Journal de Sainte-Hélène. 1815-1818, II, p. 324.

26.Le Mémorial de Sainte-Hélène, I, p. 173.

27.Barry E. O'Meara, Napoléon dans l'exil, présentation, notes et introduction de Paul Ganière, nouvelle traduction de Charles-Otto Zieseniss, Paris, Fondation Napoléon, t. I, p. 221.

28.Le Mémorial de Sainte-Hélène, I, p. 575. Déclaration identique chez O'Meara, Napoléon dans l'exil, I, 359.

29.Mémoires de Constant, premier valet de chambre de l'Empereur, Genève, éd. 1978, t. I, p. 24.

30.Chateaubriand, Mémoires d'Outre-Tombe, édition de Jean-Paul Clément, Paris, Gallimard, 1998, pp. 1179 et 1165.

31.Mémoires du général Auguste Petiet, hussard de l'Empire, texte établi par Nicole Gotteri, Paris, SPM, 1996, p. 275. 
32.Mémoires du général baron Boulart, édition de Jacques Jourquin, Paris, Tallandier, 1992, p. 76.

33.A. Aulard, " Tableau de la situation de Paris du 3 messidor an VIII », Paris sous le Consulat, Paris, 1903, t. I, p. 437.

\section{RÉSUMÉS}

Les mémoires des acteurs de l'épisode napoléonien (comme ceux de Rapp et Savary), et les écrits de Sainte-Hélène (Las Cases, Antommarchi, Gourgaud, O'Meara, Bertrand) ont contribué à rendre populaire le portrait d'un Desaix intelligent, magnanime, un des meilleurs généraux de la Révolution. Ce portrait est rehaussé par les sentiments d'amitié et d'admiration que Desaix aurait nourris à l'égard de son chef Bonaparte. Réciproquement, Napoléon a contribué à grandir la mémoire de celui qui sacrifia sa vie pour le sauver à Marengo. La légende de Desaix, général à l'antique, créée dès le Consulat, se fonde, entre autres facteurs, sur ce type de récits.

The Legend of Desaix in the Memoirs of Two of his Aides-de-camp and in the writings of Saint Helena.

The memoirs of those who took part in the Napoleonic saga (such as Rapp and Savary) and the writings of Saint-Helena (Las Cases, Antommarchi, Gourgaud, O'Meara, Bertrand) have contributed to the popular image of Desaix as intelligent, magnanimous and one of the best generals of the Revolution. This image is strengthened by the feelings of friendship and admiration Desaix is said to have nurtured towards his chief Bonaparte. Reciprocally, Napoleon helped magnify the memory of the man who sacrificed his life to save him at Marengo. The legend of Desaix, a general in the antique mould, created under the Consulate, is based in large part on this type of narrative.

INDEX

Mots-clés : Desaix, légende napoléonienne, mémoires

\section{AUTEUR}

THIERRY LENTZ

Directeur de la Fondation Napoléon 